\title{
EL AGRO CASTELLANO EN EL SIGLO XVII: ¿DEPRESION O «REAJUSTES Y READAPTACIONES»?
}

\author{
ENRIQUE LLOPIS AGELAN \\ Universidad Complutense
}

El propósito de este pequeño ensayo es replantear la discusión sobre la existencia o inexistencia de una auténtica depresión agraria en la Castilla del Seiscientos y, en su caso, sobre el alcance y los factores determinantes de aquélla.

Hasta hace pocos años, independientemente del grado de fundamentación de los juicios, podía hablarse de unanimidad, o casi completa unanimidad, en las caracterizaciones de los especialistas acerca de la evolución de la economía española durante el siglo xviI: más allá de matizaciones espaciales, temporales o sectoriales, todos reconocían la existencia de una crisis generalizada y prolongada en la España de los Austrias menores. Dos artículos de Gonzalo Anes, publicados en $1978^{\prime}$, han puesto en cuestión ese balance de la actividad productiva de nuestro Seiscientos que había sido, hasta entonces, suscrito por la inmensa mayoría de historiadores y economistas. Los textos de Anes abrían una importante polémica e incitaban a nuevas reflexiones e investigaciones sobre la trayectoria de la economía española en el siglo xvir. Quiero reiterar que mis consideraciones se circunscribirán al sector agrario castellano.

Anes sostiene que la pretendida «depresión» agraria castellana del siglo xvir consistió en una «serie de reajustes y readaptaciones que se produjeron lenta y automáticamente para armonizar la producción de subsistencias y el número de habitantes» ${ }^{2}$. Tal tesis la fundamenta en los siguientes datos y argumentos:

1. No se operó «una tendencia general a la despoblación» en dicha cen. turia.

2. Las series de diezmos de que se dispone no permiten determinar con claridad, entre otras razones, por la escasez de datos sobre las cosechas de uvas y aceitunas y sobre los esquilmos del ganado, el signo de la tendencia o tendencias de la producción agraria castellana durante el Seiscientos; no obstante,

- Gonzalo Anes (1978 a y $1978 b$ ).

2 Gonzalo Anes (1978 a), p. 100. 
del análisis de la información cuantitativa publicada cabe inferir más un cambio en la composición del producto agrario que un movimiento depresivo del mismo.

3. Ni siquiera alli donde descendió el número de activos, la producción agraria tuvo necesariamente que registrar una tendencia a la baja, dado que solía existir un importante "paro encubierto" y que la disminución de la superficie labrada permitía mejorar la calidad media de los terrenos cultivados y ampliar la cría de ganado estante. Es decir, tras la reducción de la productividad agraria y de los rendimientos medios por unidad de superficie sembrada que ocasionó el intenso proceso roturador operado durante el siglo xvI, el descenso de población pudo haber favorecido la recuperación de aquéllos.

En la primera parte de este artículo discutiré algunos de los argumentos empleados por Anes, aportaré varias series para el estudio de los movimientos de precios relativos e intentaré establecer un balance de la evolución del agro castellano en el siglo xvir.

Quedan aún por aclarar bastantes e importantes cuestiones acerca del movimiento de la población española en el Seiscientos, pero que ya ha quedado firmemente establecido que el balance global de la centuria fue de estancamiento o de ligerísima alza. Es decir, las tasas de crecimiento real o vegetativo de la población fueron nulas o muy pequeñas ${ }^{3}$. Ello me sugiere el siguiente interrogante: en el contexto de poblaciones de tipo antiguo, ies concebible que se produzca un pobre balance demográfico secular sin la concurrencia de importantes dificultades económicas? En otras palabras, ¿puede explicarse el comportamiento demográfico de la España del Seiscientos sin la existencia de una depresión económica relativamente prolongada y generalizada? El estancamiento secular del número de habitantes en poblaciones de tipo antiguo, salvo que éstas se viesen afectadas por crisis de mortalidad de

${ }^{3}$ Bustelo (1972) ha señalado que si la población española en el siglo XVII hubiese crecido a una tasa del 0,35 por 100 , «que correspondería aproximadamente a una esperanza de vida al nacer de veinticinco años», hacia 1712 debería haberse manifestado un excedente entre 2 y 3 millones de personas con respecto a la cifra que efectivamente se registró en esa fecha. La cuantía de ese excedente depende de la hipótesis de partida que elijamos. Si suponemos un estancamiento demográfico en el Seiscientos, el excedente aperdido* sería de unos 3 millones; en cambio, si admitimos un ligero crecimiento de la población, entonces el excedente se situaría en unos 2 millones. Una tasa de crecimiento del 0,35 por 100 no es nada exagerada, ya que en el siglo xvirI, pese a que las secuelas de las crisis de mortalidad, como ha demostrado Vicente Pérez Moreda (1980), fueron más intensas que en el Seiscientos, la población española, según la estimación de Livi Bacci, que los especialistas han dado por buena, creció algo por encima del 0,4 por 100 . 
enorme intensidad en extensas áreas, circunstancia que no llegó a producirse en la Europa moderna, debió ser resultado de la actuación de los controles malthusianos, tanto de los negativos como de los positivos; es más, se requiere una actuación "enérgica» de aquéllos para que el crecimiento vegetativo sea muy pequeño o nulo. Habida cuenta de que resulta muy poco probable el funcionamiento de los controles malthusianos al margen de un deterioro de la situación económica de buena parte de las poblaciones, el mero balance demográfico global del siglo constituye una evidencia que avala la tesis de la existencia de una depresión económica en la España del Seiscientos.

Por otro lado, el balance demográfico global de la centuria, como es obvio, encubre los contrastes regionales y el movimiento de población.

¿Qué sucedería si, en vez de comparar el número de habitantes de 1591 y el de 1712 , cotejásemos la población española de 1600 y la de 1650 ? Muy probablemente, el balance demográfico de esa primera mitad del siglo fuese negativo ${ }^{4}$ Obsérvese que ese saldo no corresponde al de una o dos décadas, sino al de media centuria.

Los contrastes regionales tampoco deben pasar inadvertidos. Teniendo en cuenta que la población creció en Galicia y en toda la cornisa cantábrica y que el balance demográfico de la centuria fue más desfavorable para la Corona de Castilla que para la de Aragón, parece lógico que extensas áreas del interior peninsular registrasen un estancamiento o un descenso del número de habitantes ${ }^{5}$.

Conviene plantearse por qué el balance demográfico del Seiscientos fue tan mediocre. Vicente Pérez Moreda ha contribuido decisivamente al esclarecimiento de esta cuestión ${ }^{6}$. A este respecto ha realizado las siguientes consideraciones:

1. Si las crisis generalizadas de mortalidad sólo pudieron ocasionar un pequeño porcentaje de las defunciones registradas durante la centuria, la incidencia de la mortalidad catastrófica sobre el crecimiento vegetativo debió ser bastante exigua.

2. Sin contabilizar a Galicia y a la cornisa cantábrica, las zonas que presentan un balance demográfico más favorable, la Corona de Aragón y el lito-

+ Angel García Sanz ha estimado en un 20-25 por 100 la pérdida de efectivos de la Corona de Castilla entre 1591 y 1650 (A. García Sanz (1985), p. 14).

' Como es bien conocido, salvo Madrid, las ciudades castellanas experimentaron una profunda depresión durante el siglo xvir. El balance demográfico de las zonas rurales no fue tan desfavorable, pero debieron ser muchos más los núcleos que perdieron efectivos que los que registraron incrementos de población, sobre todo si comparamos los niveles de 1600 y de 1675 . Podrían citarse numerosísimos trabajos que corroboran esta última afirmación, pero me parece suficiente con remitir a las series de bautismos que aparecen en la tesis doctoral de Vicente Pérez Moreda.

- Vicente Pérez Moreda (1980), pp. 294-326. 
ral levantino y andaluz de la Corona de Castilla, fueron, precisamente, las que se vieron afectadas por una mayor mortalidad catastrófica.

3. Pese a que las crisis de mortalidad en el Setecientos provocaron, cuando menos, efectos tan devastadores como en el Seiscientos, la población española creció en aquella centuria, probablemente, a una tasa superior al 0,4 por 100 , mientras que en ésta, en el mejor de los casos, no se superó el 0,15 por 100 .

Pérez Moreda concluye de este modo su documentado análisis sobre las razones del pobre balance demográfico de Castilla en el siglo xvir: «éstas -se refiere a las crisis de mortalidad - no fueron lo bastante frecuentes ni graves en el interior castellano a lo largo del siglo xvir como para determinar por sí mismas la evolución desfavorable, regresiva o estacionaria, de la tendencia demográfica. Debieron influir otros factores decisivos, entre los que cabe destacar la emigración" ${ }^{7}$. Ello viene a corroborar lo ya apuntado por Jordi Nadal: «En la despoblación castellana jugó una ". peste" económica peor que todas las pestes reales ${ }^{8}$.

En suma, el escaso dinamismo demográfico de Castilla durante el Seiscientos parece ser más secuela de los movimientos migratorios y de la caída de la natalidad que resultado de la acción devastadora de la mortalidad catastrófica 9 . Esta conclusión contribuye a dilucidar la polémica acerca de la existencia de una depresión económica generalizada y prolongada en la Castilla del siglo xvir, ya que las variaciones en la natalidad y los movimientos migratorios respondían, ante todo, a cambios en la coyuntura económica. Por consiguiente, si se admite que en la Castilla del Seiscientos tuvo lugar un descenso en la natalidad y una importante corriente migratoria hacia Indias y hacia otras zonas peninsulares ${ }^{10}$, difícilmente puede negarse la existencia de un prolongado movimiento contractivo durante el siglo xvir.

También resultan significativas las características de la mortalidad catastrófica que asoló a Castilla en el «siglo de la decadencia»: como ha demostrado Pérez Moreda, el papel del tifus, enfermedad estrechamente vinculada a las carencias alimenticias, tendió a ser cada vez más importante î. Por enési-

Ibidem, p. 320.

- Jordi Nadal (1971), p. 81.

- Las probables variaciones de la mortalidad ordinaria, según entiende implícitamente Pérez Moreda, no fueron lo suficientemente importantes como para explicar el movimiento de la población castellana durante el siglo Xvir.

10 La emigración por motivos políticos, la expulsión de los moriscos, no tuvo la entidad suficiente como para afectar de manera sensible al movimiento de la población castellana. Según Henri Lapeyre, salieron de Castilla y de Extremadura 44.625 moriscos, estimación que ha sido dada por buena por A. Domínguez Ortiz y Bernard Vincent (1978), p. 200.

"Vicente Pérez Moreda (1980), pp. 294-308. 
ma vez, pues, las evidencias demográficas revelan el deterioro de la economía castellana en el transcurso del Seiscientos.

En los últimos años no han aparecido muchos trabajos sobre las tendencias de la producción agraria castellana durante el siglo xVII, pero considero que nuestro conocimiento de esta cuestión ha progresado considerablemente tras la publicación de largas series decimales del inmenso Arzobispado de Toledo ${ }^{12}$. Ello me ha permitido confeccionar una serie de producción de «pan» que incluye los Arciprestazgos de Alcalá de Henares, Alcaraz, Alcolea de Torote, Brihuega, Buitrago, Calatrava, Canales, Escalona, Guadalajara, Hita, Illescas, Madrid, Montalbán, Ocaña, Rodillas, Santa Olalla y Maqueda, Talamanca, Talavera de la Reina, Zorita de los Canes y Almoguera y Vicaría de la Puebla de Alcocer ${ }^{13}$, serie que he reflejado en el gráfico adjunto (también, de manera sintética, aparece a continuación en el cuadro A).

\section{CUADRO A}

La producción de «pan» en el Arzobispado de Toledo

(Medias anuales expresadas en números índice) (*)

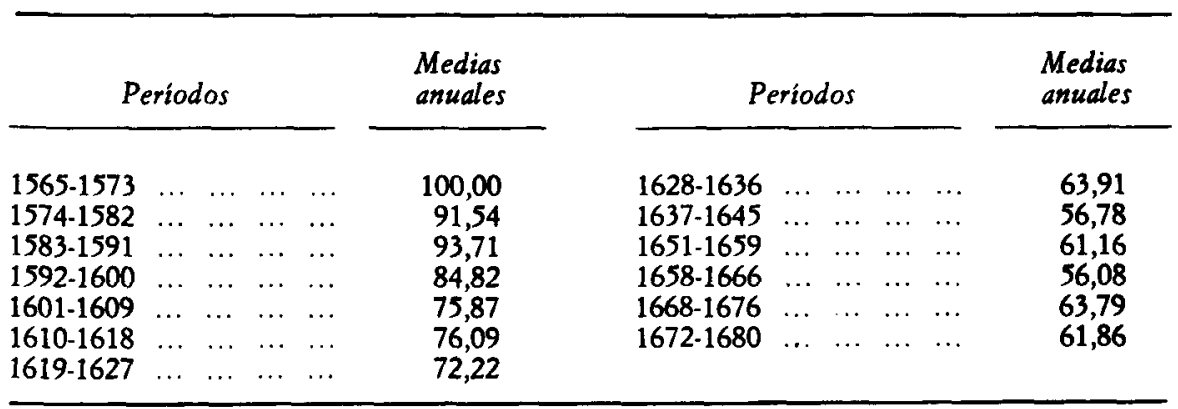

(*) He tomado como base 100 la media del período 1565-1573.

FUENTE: Elaboración propia a partir de las series de producción de «pan» en los Arciprestazgos del Arzobispado de Toledo publicadas por Jerónimo López Salazar y Manuel Martín Galán (1981), pp. 56-101.

12 J. López Salazar y M. Martín Galán (1981), pp. 56-101.

is Sólo dos Arciprestazgos del Arzobispado han quedado excluidos: el de Toledo y el de La Guardia. 
Producción de pan en el Arzobispado de Toledo

(Medias móviles de nueve años expresadas en números índice)

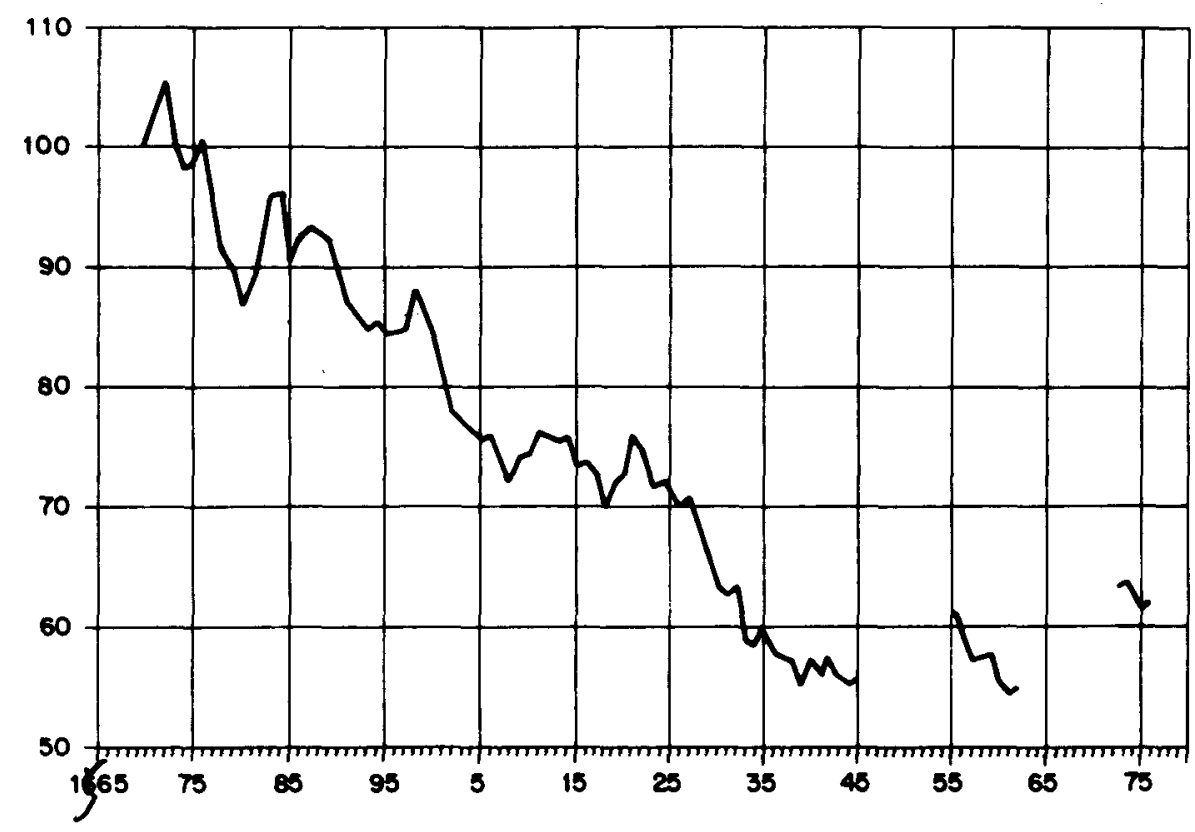

Un somero examen de las cifras ya permite efectuar tres observaciones:

1. Poco antes de concluir el tercer cuarto del siglo xvi tuvo lugar la inversión de la tendencia alcista de las cosechas de granos en el conjunto del Arzobispado de Toledo. En fecha relativamente temprana, pues, se detuvo el movimiento ascendente de la producción de cereales en gran parte de Castilla la Nueva, ya que en tierras de Segovia ${ }^{14}$ y en la Bureba ${ }^{15}$ tal fenómeno no aconteció hasta entrados los ochenta.

2. Las cosechas de granos en el Arzobispado de Toledo registraron un intenso movimiento descendente entre los últimos años del tercer cuarto del siglo xvi y finales de la década de los treinta del xvir, hasta el punto de que en esta fecha del Seiscientos la producción media anual de cereales apenas superaba la mitad de la obtenida hacia 1570.

3. Aun cuando la depresión tocó fondo a finales de los treinta, una verdadera inversión de la tendencia no tuvo lugar hasta poco antes de los seten-

14 Angel García Sanz (1977), pp. 105-106.

1s Francis Brumont (1984), pp. 145-150. 
ta; además, el titubeante movimiento ascendente sólo logró recuperar, al menos hasta 1680 , poco más de la octava parte del terreno perdido con respecto al máximo alcanzado a comienzos de los setenta del siglo xvi.

Vale la pena realizar un análisis más detenido del persistente movimiento depresivo. En él pueden distinguirse cinco fases:

a) En la primera, situada entre mediados de los setenta y de los ochenta del siglo xvi, el nivel medio de las cosechas de grano descendió en torno al 13 por 100 .

b) La segunda, que transcurre entre mediados de los ochenta y primeros años del siglo xvir, se caracteriza por una fugaz recuperación, gracias a las buenas cosechas de 1585,1586 y 1587, que dio paso a un estancamiento de la producción de cereales a un nivel medio parecido al de finales de la primera fase.

c) En la tercera, que comprende los años centrales del primer decenio del Seiscientos, se opera una nueva e intensa caída de las cosechas, situándose el nivel medio cerca de un 18 por 100 por debajo del de la fase precedente.

d) El estancamiento en el escalón productivo anterior constituye el rasgo más sobresaliente de las dos décadas que separan los años finales del primer decenio del siglo xvil de los últimos del tercero.

e) Los treinta, al igual que en otras zonas, resultaron dramáticos para Castilla la Nueva: partiendo de un peldaño muy bajo, el nivel medio de las cosechas de granos descendió más de un 20 por 100.

En suma, en el Arzobispado de Toledo, los períodos claves del movimiento contractivo fueron, por orden de intensidad, los años treinta del Seiscientos, la parte central del primer decenio de esta centuria y la década que transcurrió entre mediados de los setenta y de los ochenta de la precedente.

En el cuadro B he sintetizado los escasos y fragmentarios datos que he logrado reunir sobre la trayectoria de la producción de granos en la Ciudad y Tierra de Trujillo.

Aunque la información resulta insuficiente para nuestro propósito, merecen destacarse algunos fenómenos:

a) Tanto en 1550 como en 1560 se cosecharon más del doble del total de granos que se recogieron, en promedio, entre 1711 y 1734, mientras que este último nivel únicamente no llegó a alcanzarse en 1556, siendo el «déficit» de sólo un 12,91 por 100 .

b) Resulta aún más significativo que en ningún año del período 17111734 se llegasen a recolectar el 80 por 100 de los cereales cosechados en 1550 . 


\section{CUADRO B}

\section{Tercias Reales de la Ciudad y Tierra de Trujillo \\ (En fanegas)}

\begin{tabular}{|c|c|c|c|c|}
\hline$A \tilde{n} o s$ & Trigo & Cebada & Centeno & Total de granos \\
\hline $\begin{array}{llllll}1550 & \ldots & \ldots & \ldots & \ldots & \ldots \\
1556 & \ldots & \ldots & \ldots & \ldots & \ldots \\
1559 & \ldots & \ldots & \ldots & \ldots & \ldots \\
1560 & \ldots & \ldots & \ldots & \ldots & \ldots\end{array}$ & $\begin{array}{l}2.943,58 \\
1.214,04 \\
1.562,33 \\
3.148,68\end{array}$ & $\begin{array}{l}688,62 \\
299,31 \\
278,58 \\
552,37\end{array}$ & $\begin{array}{r}403,16 \\
109,31 \\
77,72 \\
185,45\end{array}$ & $\begin{array}{l}4.035,36 \\
1.622,66 \\
1.918,63 \\
3.886,50\end{array}$ \\
\hline & \multicolumn{4}{|c|}{ MEDIAS ANUALES } \\
\hline Periodo & Trigo & Cebada & Centeno & Total de granos \\
\hline $\begin{array}{cccc}1711-1734 & \ldots & \ldots & \ldots\end{array}$ & 904,82 & 360,16 & 598,31 & $1.863,29$ \\
\hline
\end{tabular}

Fuentes: «Hojas de Gracias», Archivo del Monasterio de Guadalupe, leg. 143; «Hojas de Pan», AMG, leg. 149.

Si consideramos únicamente el trigo, dicho porcentaje desciende hasta el 60 por 100.

c) Los pesos específicos de la cebada y, sobre todo, del centeno, dentro de la producción de cereales, se incrementaron de manera sustancial entre mediados del siglo xvi y el primer tercio del siglo xvirr. La parcial sustitución de bueyes por mulas, aun cuando en la provincia de Cáceres este proceso no parece alcanzar sus momentos decisivos hasta después de $1850^{16}$, pudo ser la causa del aumento de la importancia relativa de la cebada. El caso del centeno debió estar relacionado con la necesidad de poner en cultivo terrenos de inferior calidad, lo que pudo venir determinado por los adehesamientos y acotamientos de términos públicos y por la extensión de las posesiones mesteñas.

En suma, el nivel medio de la producción de granos debía ser bastante más elevado en la sexta década del siglo xvi que en el primer tercio del siglo xvill; además, durante la segunda mitad del Quinientos y el Seiscientos se registró un apreciable cambio en la importancia relativa de los distintos

\footnotetext{
7* En la provincia de Cáceres, todavía en 1891, las yuntas de ganado vacuno suponían 1 62,5 por 100 del total (Dirección General de Agricultura, Industria y Comercio, La gahaderia en España. Avance sobre la riqueza pecuaria en 1891, formado por la Junta Consuttlva Agronómica conforme a las memorias reglamentarias que en el citado año ban redactado los Ingenieros del Servicio Agronómico, Madrid, 1892, tomo IV, p. 35). Santiago Zapata me facilitó generosamente esta referencia.
} 
cereales que parece apuntar hacia un empeoramiento de la dieta, al menos en la parte de ésta integrada por aquéllos. Resulta, pues, bastante probable que también la zona de Trujillo se viese afectada por una intensa depresión agrícola en el siglo xvir, aun cuando poco o nada podemos señalar sobre la forma y características de este movimiento contractivo.

Pese a que las tendencias de las cosechas de granos presentan peculiaridades cronológicas en las distintas zonas, también en tierras de Segovia, Palencia y Toledo, como han mostrado A. García Sanz, Alberto Marcos y Michael Weisser ${ }^{17}$, respectivamente, la producción cerealícola registró una intensa depresión durante buena parte del Seiscientos. Por tanto, con la información que hoy manejamos, difícilmente puede cuestionarse el persistente movimiento recesivo de las cosechas de granos durante la primera mitad del siglo xviI en extensas áreas de las dos Castillas y de Extremadura, siendo probable que la producción cerealícola hubiese descendido más de un 30 por 100 entre 1565-1574 y 1640-1649. La extrema lentitud del movimiento de recuperación fue tan significativa, cuando menos, como el anterior hundimiento de las can. tidades recolectadas de granos; así lo evidencia el hecho de que a finales del siglo XVII, en numerosas zonas castellanas, aún no se hubiese recobrado el nivel que habían alcanzado las cosechas de cereales al concluir el tercer cuarto de la centuria anterior.

\section{III}

Desde un punto de vista teórico, el descenso de las cantidades recolectadas de granos no tuvo por qué implicar una caida del producto agrario, ya que aquél pudo ser compensado con un alza de los esquilmos del ganado y de otras cosechas, especialmente de las de uvas y aceitunas. Gonzalo Anes ha señalado que el comportamiento de los precios relativos durante la primera mitad del siglo xvir determinó un aumento del coste de oportunidad de dedicar la tierra al cultivo de cereales, lo que, al coincidir con la despoblación de algunas zonas rurales, hubo de favorecer la expansión de la ganadería ${ }^{18}$.

A través del examen de varias series de precios relativos intentaré determinar, aunque sólo sea grosso modo, en qué medida las modificaciones de los términos de intercambio pudieron estimular reestructuraciones en el producto agrario castellano en la primera mitad del Seiscientos.

Comenzaré con un somero análisis de los precios relativos de los granos. En el cuadro $C$ presento una síntesis de la evolución de los términos de intercambio del trigo y de la cebada en Castilla la Nueva y León.

17 Angel García Sanz (1977); Alberto Marcos (1985); Michael Weisser (1973).

18 Gonzalo Anes (1978 a), p. 93; íd. (1984), p. 9. 


\section{CUADRO C}

\section{Precio del trigo / Precio de la cebada}

(Medias anuales)

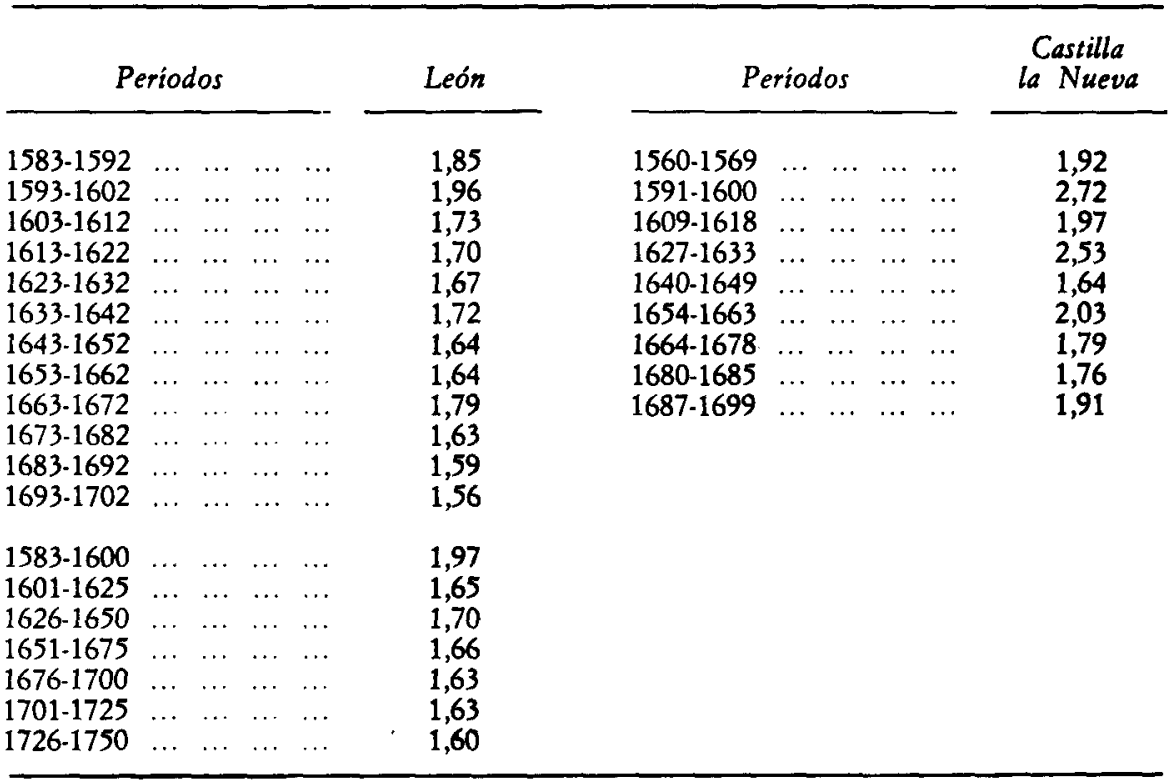

FuENTES: La serie de León la he elaborado con los precios medios anuales del trigo y de la cebada vendidos por el Monasterio de Sandoval, que me han sido facilitados generosamente por José Antonio Sebastián Amarillas, quien los recogió de los Libros de Caxa, AHN, clero, libros 5169 y 5174-76; la de Castilla la Nueva la he confeccionado a partir de las publicadas por E. J. Hamilton para el trigo y la cebada 19 .

Los años finales del siglo Xvi no constituyen un adecuado punto de referencia para examinar la trayectoria de los precios relativos agrarios en la siguiente centuria, ya que aquéllos fueron un período en el que la carestía de los cereales panificables alcanzó cotas excepcionales. Teniendo presente este extremo, las cifras recogidas en el cuadro $C$ muestran la gran estabilidad de los términos de intercambio de los dos principales granos, en el mercado leonés, durante el siglo xviI y primera mitad del xvirr. En lo referente a Castilla la Nueva, si tomamos como base para la comparación los años sesenta del siglo XVI, tampoco los datos evidencian una apreciable depreciación del trigo

${ }^{19}$ E. J. Hamilton (1934) -utilizo la versión en castellano-, pp. 358-365 y 388-393; id. (1947), pp. 234-241. 
en términos de cebada durante la primera mitad del Seiscientos. Consiguientemente, el comportamiento de los precios relativos no parece que pudiese constituir un importante estímulo para la sustitución del cultivo del trigo por el de la cebada en el transcurso del movimiento contractivo.

En el cuadro $\mathrm{D}$ he sintetizado la tendencia de los términos de intercambio del trigo y del vino en La Rioja y Castilla la Nueva.

\section{CUADRO D}

Precios del trigo / Precios del vino (Medias anuales expresadas en números índice) (*)

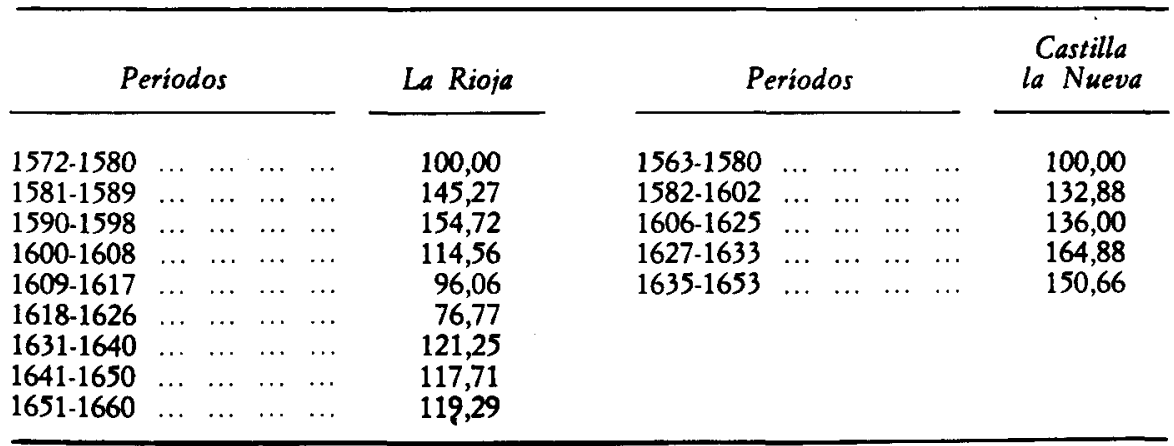

(*) He tomado como base 100 la media de los años 1572-1580, en el caso de La Rioja, y la de los años 1563-1580, en el de Castilla la Nueva.

Fuentes: La serie de La Rioja la he elaborado con los precios medios anuales del trigo y vino vendidos por el Monasterio de La Estrella (AHN, clero, libros 5966, 5978 y 6000); la de Castilla la Nueva la he construido con las publicadas por E. J. Hamilton para el trigo y el vino.

Si diésemos por buena la serie de precios del vino que proporciona Hamilton para Castilla la Nueva, calificativo que no debe otorgársele hasta que no se realicen las oportunas contrastaciones, tendríamos que admitir que, en dicha región, la tendencia de los precios relativos no sólo no justificó la plantación de vides en tierras de epan llevar» durante la primera mitad del siglo xviı, sino que alentó justamente lo contrario.

La serie de precios del vino de La Rioja es mucho más fiable, ya que en ella se relacionan las cotizaciones alcanzadas en el mercado por un producto homogéneo: los caldos obtenidos en las tierras del Monasterio de La Estrella. En La Rioja, de acuerdo con los datos recogidos en el cuadro D, parece que 
sólo en el período 1618-1628 el comportamiento de los términos de intercambio alentó de manera sensible las plantaciones de vides a costa de los terrenos de labor. Por tanto, en la primera mitad del Seiscientos, el movimiento de precios relativos no pudo ser causante de un cambio significativo en la composición del producto agrario tendente a incrementar la participación del vino a costa de la de los cereales.

El trigo tendió a encarecerse en términos de aceite entre, aproximadamente, 1560 y 1650 en Castilla la Nueva. Dicha apreciación, como puede comprobarse en el cuadro $E$, fue de algo más de un 60 por 100 entre 1563-1580 y 1635-1653. En consecuencia, el movimiento de precios relativos no parece que pudiese estimular nuevas plantaciones de olivares en terrenos de labor durante la primera mitad del siglo xvir.

\section{CUADRO E}

Precios del trigo / Precios del aceite

(Medias anuales expresadas en números índice) $(*)$

\begin{tabular}{|c|c|c|c|c|}
\hline Períodos & & $\begin{array}{l}\text { Castilla } \\
\text { la Nueva }\end{array}$ & Períodos & $\begin{array}{l}\text { Castilla } \\
\text { la Nueva }\end{array}$ \\
\hline $\begin{array}{lll}1563-1580 & \ldots & \ldots \\
1582-1602 & \ldots & \ldots \\
1606-1625 & \ldots & \ldots \\
1627-1633 & \ldots & \ldots\end{array}$ & $\begin{array}{ll} & \cdots \\
\cdots & \cdots \\
\cdots & \cdots \\
\cdots & \cdots\end{array}$ & $\begin{array}{l}100,00 \\
131,50 \\
154,79 \\
147,94\end{array}$ & $\begin{array}{lllll}1635-1653 & \ldots & \ldots & \ldots & \ldots \\
1655-1685 & \ldots & \ldots & \ldots & \ldots \\
1687-1699 & \ldots & \ldots & \ldots & \ldots\end{array}$ & $\begin{array}{r}160,27 \\
112,32 \\
97,26\end{array}$ \\
\hline
\end{tabular}

(*) He tomado como base 100 la media de los años 1563-1580.

Fuentes: Earl J. Hamilton, El tesoro americano y la revolución de los precios en España, 1501-1650, Barcelona, 1975, pp. 360-365 y 388-393; del mismo autor, War and Prices in Spain, 1651-1800, Cambridge (Mass.), Harvard University Press, 1947, pp. 238-241.

Descartadas las otras opciones, ¿pudo la ganadería compensar el movimiento contractivo de las cosechas de cereales? Durante los primeros decenios del siglo xvil, el número de cabezas trashumantes, según las estimaciones de Jean-Paul Le Flem ${ }^{20}$, no tendió a aumentar, registrándose frecuentemente cifras que no llegaban a representar el 60 por 100 de las de los máximos de la primera mitad del Quinientos y de la segunda mitad del Setecientos. Apuntan en esa misma dirección las conclusiones a las que llegué tras un minucioso examen de las cuentas de la cabaña trashumante del Monasterio de

\footnotetext{
20 Jean.Paul Le Flem (1972), pp. 68-70.
} 
Guadalupe ${ }^{21}$ : en los años finales del siglo xvi y en las cuatro primeras décadas del xviI, las explotaciones mesteñas debieron registrar una profunda crisis como consecuencia del fortísimo encarecimiento de los pastos invernales y de la relativa debilidad de la demanda exterior de lana, agravada en algunos períodos, como ha mostrado Jonathan I. Israel ${ }^{22}$, por las dificultades que los conflictos bélicos ocasionaban al comercio exterior. Los datos que presento en el cuadro $\mathrm{F}$ confirman lo anteriormente expuesto: los términos de intercambio no fueron favorables para los ganaderos trashumantes en los cuatro primeros decenios del Seiscientos ${ }^{23}$.

\section{CUADRO F}

\section{Precios relativos}

(Medias anuales expresadas en números índice) $\left({ }^{*}\right)$

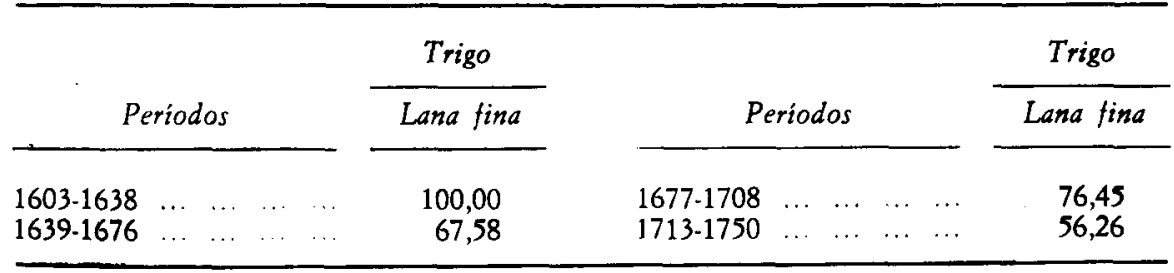

(*) He tomado como base 100 la media de los años 1603-1638.

FUENTES: He utilizado los precios medios anuales del trigo vendido por el Monasterio de Sandoval, de León, y las cotizaciones de la pila de lana fina del Cabildo de Segovia (éstas me fueron gentilmente facilitadas por A. García Sanz, quien las transcribió de los Libros de Menudos del Archivo Capitular de Segovia).

Sólo resta, pues, una alternativa: únicamente un hipotético crecimiento de las cabañas estantes podría haber impedido que la depresión cerealícola conllevase un acusado descenso del producto agrario castellano en los cuatro primeros decenios del Seiscientos.

Es cierto, como señala Gonzalo Anes ${ }^{24}$ y puede constatarse en el cuadro $G$, que, en Castilla la Vieja, el precio de la carne tendió a aumentar con mayor intensidad que el del trigo durante la primera mitad del siglo xvir.

${ }^{21}$ Enrique Llopis (1980), pp. 125.168.

22 Jonathan I. Israel (1980), pp. 193-211.

${ }^{23} \mathrm{Si}$ hubiese utilizado una serie de precios del trigo de Castilla la Nueva o Extremadura, los términos de intercambio resultarían aún más desfavorables para los mesteños en el primer tercio del siglo XviI.

${ }_{24}$ Gonzalo Anes (1978 a), p. 93; id. (1979), p. 287. 
Ahora bien, si tomásemos como punto de referencia los términos de intercambio de los años setenta del Quinientos, la trayectoria de aquéllos no se presenta tan favorable para los productores de carne. Además, ese comportamiento de los precios relativos no era condición suficiente para que apareciesen importantes estímulos para ampliar la cría de ganados, ya que un acusado crecimiento de los gastos de explotación podía determinar un descenso de los beneficios de los dueños de cabañas. Nos consta que la superficie de pastos comunales, debido a las roturaciones, adehesamientos, acotamientos y plantaciones de viñedo, registró un brusco descenso en la segunda mitad del siglo xvi y, muy probablemente, en el primer tercio del xvir. Ello determinó, como denunciaron los contemporáneos ${ }^{25} \mathrm{y}$ he podido constatar en las cuentas de las cabañas del Monasterio de Guadalupe ${ }^{26}$, un intensísimo movimiento

\section{CUADRO G}

\section{Precio del trigo / Precio de carne}

(Medias anuales expresadas en números índice) $\left({ }^{*}\right)$

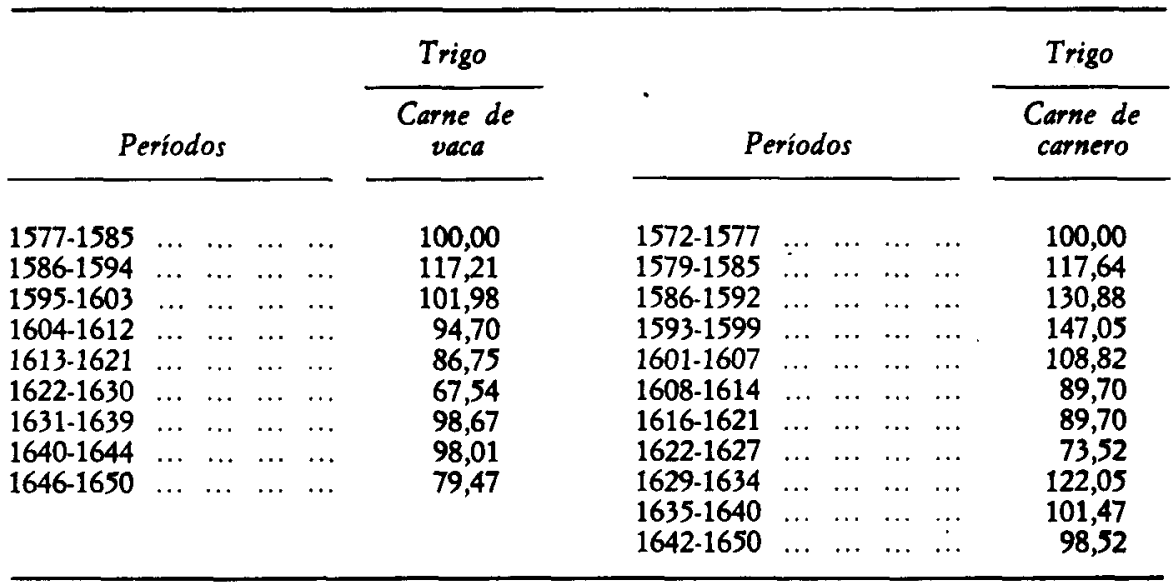

(*) He tomado como base 100 la media de los años $1577-1585$, en el caso de la carne de vaca, y la de los años 1572-1577, en el de la carne de carnero.

FuENTES: Las series las he formado con los precios medios anuales del trigo vendido por el Monasterio de La Estrella y con los publicados por E. J. Hamilton para la carne de vaca y de carnero en Castilla la Vieja.

${ }^{25}$ Miguel Caxa de Leruela (1631), pp. $105-106$.

to Entre 1515-1524 y 1628-1637, el precio del trigo en Castilla la Nueva, de acuerdo con la serie publicada por Hamilton, se multiplicó por 5,54, y el de los pastos del Monasterio de Guadalupe por 8,33 (Enrique Llopis (1980), p. 144). 
ascendente del precio de las hierbas entre, aproximadamente, 1550 y 1630. Teniendo en cuenta que éstas representaban un elevado porcentaje de los costes de producción de las explotaciones ganaderas, resulta bastante probable que los balances de las cabañas estantes no sólo no mejorasen durante la primera mitad del Seiscientos, sino que tendieran a empeorar.

Considerando que la demanda de carne hubo de hundirse en la Castilla la Vieja de la primera mitád del siglo xvir, debido al descenso de población, a la profunda crisis de las ciudades y a las dificultades económicas en general, desde mi punto de vista, el encarecimiento de aquélla constituye, ante todo, un elocuente testimonio de la depresión de la ganadería estante. Según Caxa de Leruela, «de los ganados que llaman estantes, que solían ser cuatro veces más, que los transhumantes (o trasterminantes), faltan de cuatro partes las tres ${ }^{27}$. No hay duda de que el alcalde mayor entregador exageraba el desastre ${ }^{28}$, pero resulta difícil cuestionar la existencia de una aguda crisis de las cabañas estantes si se tiene presente el grave problema de los pastos y el comportamiento del precio de la carne en un período en el que la demanda de este artículo tuvo que experimentar un intenso movimiento contractivo.

En Extremadura, los términos de intercambio entre el trigo y las distintas especies ganaderas, según puede apreciarse en el cuadro $\mathrm{H}$, se comportaron

\section{CUADRO $\mathrm{H}$}

Precio de cabezas de ganado / Precio del trigo (Medias anuales expresadas en números índice) $(*)$

\begin{tabular}{|c|c|c|c|c|}
\hline & $\begin{array}{l}\text { Novillos } \\
\text { de tres } \\
\text { años }\end{array}$ & $\begin{array}{l}\text { Ovejas } \\
\text { estantes }\end{array}$ & & Yeguas \\
\hline Periodos & Trigo & Trigo & Periodos & Trigo \\
\hline $\begin{array}{llll}1598-1612 & \ldots & \ldots \\
1628-1652 & \ldots & \ldots \\
1653-1677 & \ldots & \ldots\end{array}$ & $\begin{array}{r}100,00 \\
72,36 \\
132,70\end{array}$ & $\begin{array}{l}100,00 \\
100,00 \\
150,87\end{array}$ & $\begin{array}{lllll}1597-1612 & \ldots & \ldots & \ldots & \ldots \\
1628-1652 & \ldots & \ldots & \ldots & \ldots \\
1659-1677 & \ldots & \ldots & \ldots & \ldots\end{array}$ & $\begin{array}{r}100,00 \\
69,05 \\
94,96\end{array}$ \\
\hline
\end{tabular}

(*) He tomado como base 100 la media de los años 1598-1612.

Fuente: Hojas de Ganados, Cuentas de las Cabañas del $\ddot{M}$ onasterio de Guadalupe, Archivo del Monasterio de Guadalupe, leg. 127.

${ }^{27}$ Miguel Caxa de Leruela (1631), p. 49.

- Resulta revelador que Caxa de Leruela, pese a pertenecer a una familia de ganaderos trashumantes y a haber desempeñado un importante cargo en la Mesta, pusiese el principal énfasis en el hundimiento de la ganadería estante. Ello, obviamente, no puede considerarse como prueba inequívoca de su independencia y objetividad, pero debe tenerse en cuenta cuando se juzgue el grado de fiabilidad de los testimonios que nos presenta en su obra. 
de manera diferente que en Castilla la Vieja: los precios relativos, salvo en el caso de las ovejas, evolucionaron favorablemente para los granos, cuando menos ${ }^{29}$, durante los treinta y cuarenta del Seiscientos, período en el que la depresión cerealícola parece tocar fondo. El encarecimiento del trigo en términos de ganado vacuno y caballar, cuando el movimiento contractivo alcanza su cénit, pudo deberse a la aguda escasez de cereales panificables y/o a la fortísima caída de la demanda pecuaria y al declive menos pronunciado de la oferta de este subsector en relación a otras regiones peninsulares. Por tanto, cualesquiera que fuesen los motivos, en la Extremadura de la primera mitad del siglo xvir no parecen existir incentivos para sustituir labranza por cría de ganado, máxime si tenemos presente que los precios de los pastos registraron un vertiginoso crecimiento en las décadas finales del Quinientos y primeras
del Seiscientos.

En definitiva, los movimientos de precios relativos, debido a su sentido, en unos casos, o a su escasa entidad y/o duración, en otros, no parece que hubieran sido capaces de evitar que la depresión cerealícola se tradujese en una sensible caída del producto agrario castellano en las cuatro primeras dépastos comunales determinó que la tendencia decreciente de las cosechas de Es cierto que ésta acabaria recuperándo retroceso de la ganadería estante. tal y como que ésta acabaría recuperándose, pero tal proceso debió iniciarse, tal y como apunta Pérez Moreda para tierras segovianas ${ }^{30}$, después de que la
depresión agrícola hubiese tocado fondo.

Desde mi punto de vista, hay un argumento aún más relevante que cuestiona la hipótesis de que la expansión de las cabañas y de otros cultivos pudiese haber compensado el declive de las cosechas de cereales: cuando la venta y aprovechamiento de buena parte del terrazgo estaban sometidos a importantes restricciones, cuando sólo una pequeña porción del producto agrario se destinaba al mercado, cuando un elevado porcentaje de los cultivadores directos carecía de medios para readaptar sus explotaciones, y cuando la mayoría de los campesinos seguían preocupados, ante todo, por las cosechas de granos pa-
nificables a fin de evitar el agravamiento de sus carencias alimenticias, los cambios en los precios relativos únicamente podian provocar modificaciones
bastante limitadas en la asignación de recursos.

Dicho de otro modo, en la economía castellana del Antiguo Régimen resulta muy poco probable que alteraciones en los términos de intercambio ocasionasen cambios sustanciales en la composición del producto agrario. El propio Gonzalo Anes, tras el examen de las Relaciones para la descripción e

\footnotetext{
Nada puedo decir del período 1613-1627, ya que faltan las cuentas de las cabañas
de esos años.

${ }^{30}$ Vicente Pérez Moreda (1978), pp. 296-297.
} 
historia de los pueblos de España, mandadas confeccionar por Felipe II, reconoce que «los lugares en los que predominaba el cultivo de trigo, el de la vid o el olivo fueron muy pocos durante el siglo xvi. La diversificación de cultivos se mantuvo, en España, hasta nuestros días, en cada localidad, para asegurar un abastecimiento que no hacía posible el mercado, ya que el poder de compra de los campesinos era bajo y habían de tender a producir casi todo lo que necesitaban para su consumo" ${ }^{31}$.

\section{IV}

Una vez expuestos algunos de los datos y argumentos que me han llevado a mantener la tesis de la existencia de una intensa depresión agraria en la Castilla de la primera mitad del Seiscientos, en las líneas que siguen efectuaré algunas consideraciones sobre los factores determinantes de aquélla. Se trata, obviamente, de una mera aproximación a este amplio y complejo problema.

Si tenemos en cuenta que, en España, entre comienzos del siglo xvi y finales del $\mathbf{x I X}$, sin haberse operado cambios sustanciales en las técnicas agrarias, la población y la superficie labrada hubieron de multiplicarse por más de dos, resulta inadmisible la utilización de los esquemas malthusianos como pieza única o principal de los modelos explicativos de los cambios de tendencia a largo plazo del producto agrario durante dichas centurias. Ello no impide reconocer que determinadas áreas, en períodos concretos, han registrado crisis de carácter fundamentalmente malthusiano, como parece acontecer, según ha mostrado F. Brumont ${ }^{32}$, en tierras de la Bureba a finales del siglo xvi. Sin embargo, en Castilla la Nueva y Extremadura - $e$ incluso en bastantes comarcas de Castilla la Vieja - regiones de escasa densidad demográfica que continuaron repoblándose entre los siglos xvi y xix, deberían dejar de emplearse las limitaciones intrínsecas de los recursos agrarios como el argumento principal que, en última instancia, da cuenta de los persistentes movimientos depresivos de la producción. En consecuencia, si se quiere hacer inteligible la trayectoria del agro castellano, resulta inexcusable el análisis de las modificaciones operadas en la forma de explotación del terrazgo, modificaciones que no estaban predeterminadas, sino que fueron resultado, al menos en buena medida, de las específicas vicisitudes históricas de las regiones objeto de estudio.

Desde mi punto de vista, la depresión económica_de Castilla tuvo una estrecha relación con los cambios sustanciales que se registraron en los modos de aprovechamiento de los recursos agrarios durante las décadas finales del

${ }^{31}$ Gonzalo Anes (1978 b), p. 101.

${ }^{32}$ Francis Brumont (1984), pp. 145-150. 
siglo xvi y la primera mitad del xvir. Tales alteraciones contribuyeron decisivamente al proceso de pauperización de un elevado porcentaje de cultivadores directos al reducir de manera sensible la superficie de labor y, sobre todo, de pastizales, a las que aquéllos podían acceder de balde o satisfaciendo una cantidad simbólica. Estas afirmaciones me obligan, pues, a examinar las conexiones entre el empobrecimiento de las economías campesinas y las modificaciones operadas en el uso y distribución del terrazgo.

Aun cuando las tintas hayan sido cargadas en exceso, las siguientes palabras de David E. Vassberg constituyen un buen punto de referencia inicial: «La evidencia disponible muestra que el sistema comunitario, en sus múltiples formas, estaba en pleno apogeo en Castilla a mediados del siglo xvi. Las tierras comunitarias y baldías sostenían la ganadería y la agricultura, y constituían la clave de la estructura social y económica de la vida rural» ${ }^{33}$. El campesino, pues, no sólo solía sustentar sus rebaños en los pastos comunales, sino que frecuentemente realizaba una parte o la totalidad de sus labores en terrenos públicos. La magnitud de las ventas de baldíos realizadas durante el reinado de Felipe II pone de manifiesto la importancia que entonces tenía la labranza en aquéllos, ya que un elevado porcentaje de las tierras enajenadas habían sido previamente roturadas y cultivadas.

A medida que avanzaba la segunda mitad del Quinientos tendió a empeorar la situación de numerosos productores agrarios castellanos. Casi todos ellos hubieron de hacer frente a un aumento de la renta de la tierra y a la creciente presión fiscal. Muchos, también, se vieron negativamente afectados por la progresiva escasez de pastos para sustentar el ganado auxiliar de labranza - debido a las roturaciones y al laboreo más frecuente del terrazgo-, por el agotamiento de los suelos ante la necesidad de obtener un mayor número de cosechas, por la puesta en cultivo de terrenos de inferior calidad y por las intensas fluctuaciones del precio de los granos en las dos últimas décadas de la centuria. El alto coste de las roturaciones, como oportunamente ha destacado Gonzalo Anes ${ }^{34}$, forzaba a los campesinos a intentar asegurarse el derecho exclusivo para explotar los terrenos que habían roturado, pero también, en ocasiones, impedía que aquéllos pudiesen recurrir a este arbitrio para tratar de atender las mayores necesidades alimenticias, lo que no les dejaba otra alternativa que la intensificación de las labores que venían realizando, aun cuando este cambio no fuese habitualmente acompañado de mejoras en el cultivo y de la aplicación de mayores cantidades de abono por unidad de superficie labrada. El resultado no podía ser otro que el progresivo agotamiento de los suelos y el movimiento decreciente de las cosechas.

Ahora bien, pese a que ya parece haberse operado la inversión de la ten-

33 David E. Vassberg (1983), p. 53.

3 Gonzalo Anes (1984), p. 4. 
dencia en el conjunto del agro castellano, éste no registró un rápido hundimiento en las décadas finales del Quinientos; es más, la actuación de los mecanismos de equilibrio de este tipo de economías, especialmente los controles malthusianos, podían hacer concebir esperanzas de una pronta paralización del movimiento depresivo. Sin embargo, las tendencias contractivas no sólo no cesaron, sino que se recrudecieron. Esta singular trayectoria fue consecuencia, al menos en buena medida, de algo ya apuntado: los profundos cambios llevados a cabo en el aprovechamiento del terrazgo, principalmente en el que hasta entonces había tenido un carácter público.

Disponemos de cuantificaciones precisas sobre la elevación de los encabezamientos y de las cargas tributarias en la Castilla de la segunda mitad del siglo xvi. En 60 pueblos de la comunidad de villa y tierra de Sepúlveda, la cantidad a satisfacer por vecino para el pago de tercias y alcabalas se multiplicó por más de seis entre 1561 y $1584^{35}$. En la Bureba el alza fue menos intensa: el importe a satisfacer se multiplicó por 2,1 de 1561 a $1579^{36}$. Ahora bien, en un contexto de estancamiento o retroceso económico y demográfico, la escalada fiscal no podía proporcionar al Estado los enormes recursos que éste precisaba para seguir desarrollando su frenética actividad en el exterior. Para incrementar sustancialmente las recaudaciones en la Corona de Castilla, sin introducir cambios significativos, de hecho, en el sistema tributario -que, dicho sea de paso, fueron abortados por las oligarquías locales y los estamentos privilegiados, como así lo evidencia la desnaturalización de los millones, impuesto que, destinado a gravar la riqueza, acabó siendo sufragado en buena medida por los consumidores a través del establecimiento de sisas sobre distintos artículos ${ }^{37}$ - , la Hacienda tuvo que recurrir a enajenar parte del patrimonio territorial público y a otorgar facultades para que los municipios pudiesen vender, pignorar, acotar o arrendar algunas de sus respectivas propiedades. Angel García Sanz ya apuntó este fenómeno en uno de los más sugerentes artículos publicados en los últimos años: «El aparato del Estado utilizará, a partir de mediados del siglo XVI, numerosas vías para absorber recursos del medio rural, pero la mayor parte de ellas desembocarán al final, después de tortuosos vericuetos, a veces, en los bienes comunales: la venta, la pignoración o privatización de su aprovechamiento, solía presentarse como la fórmula mágica para corresponder con la Hacienda sin dañar los bolsillos privados, sobre todo los bolsillos de los poderosos» ${ }^{38}$.

Es cierto que las ventas de baldíos no introdujeron modificaciones sustanciales en el aprovechamiento de los recursos agrarios, dado que la mayor parte

35 Angel García Sanz (1980), p. 114.

36 Francis Brumont (1984), pp..-192-193.

${ }^{37}$ Felipe Ruiz Martín (1978), pp. 43-45.

3* Angel García Sanz (1980), p. 113. 
de las tierras enajenadas ya habían sido roturadas y puestas en cultivo. No obstante, hay que tener en cuenta que tales operaciones significaban una merma irreversible del patrimonio territorial público; además, los compradores no sólo solían obtener licencia para labrar las parcelas adquiridas, sino que no fue infrecuente que se les concediese autorización para cercarlas, lo que implicaba una disminución de los aprovechamientos colectivos en barbecheras y rastrojeras.

Probablemente, los mayores cambios en el aprovechamiento de los terrenos se produjeron a raíz de que proliferasen, desde el establecimiento del nuevo impuesto de millones, las licencias regias que autorizaban a los pueblos a romper, enajenar, pignorar, acotar y arrendar sus respectivos patrimonios. Parece, pues, alcanzarse un importante pacto, más o menos explícito, bajo la atenta mirada y aquiescencia del clero y la nobleza, entre quienes regentaban el poder municipal y la Monarquía: ésta obtenía recursos adicionales que le resultaban imprescindibles para seguir desarrollando su activa política exterior, en tanto que el clero, la nobleza y los «poderosos» locales conseguían evitar el impacto que hubiera supuesto para sus economías la estricta aplicación del nuevo impuesto de millones. Consiguientemente, la base del acuerdo parece residir en el compromiso a recaudar unas cantidades adicionales a cambio de la traslación de la carga tributaria, pero el alcance del mismo fue mucho más allá del de una simple operación de repercusión de un impuesto, ya que acabó afectando a las condiciones en las que numerosísimos productores directos accedían a recursos agrarios vitales para sus economías. Los «poderosos» locales debieron ser los grandes beneficiarios del pacto: laś concesiones regias creaban un marco favorable para la ampliación de sus haciendas, aspecto nada intrascendente en una economía en la que la oferta de tierras solía ser muy pequeña, y para participar en lucrativos negocios relacionados con la deuda municipal, al tiempo que, directa o indirectamente, les otorgaban facultades para asignar y distribuir con mucha mayor libertad los impresionantes recursos de los patrimonios concejiles. Pese a las resistencias que debieron protagonizar los campesinos menos favorecidos, los cambios que estaban acaeciendo en la administración de los bienes públicos colocaban a las oligarquías locales en una situación muy ventajosa para la eficaz defensa de sus intereses como demandantes de mano de obra, como rentistas, como ganaderos y como vendedores de excedentes agrícolas: arguyendo la urgencia de allegar fondos al municipio y amparándose en las facultades regias, ahora disponían de un mayor margen para reasignar importantes recursos agrarios de acuerdo con las características y necesidades de sus explotaciones. El ascenso económico y social de los "poderosos» locales estuvo, pues, ligado al definitivo afianzamiento en el control ejercido por aquéllos sobre el uso del 
terrazgo público a raíz de las frecuentes licencias que la Corona concedió a los pueblos con motivo de la periódica renovación de los millones.

Parece ser que, inicialmente, bastantes municipios recurrieron a los censos hipotecarios para hacer frente a las exacciones estatales. Sin embargo, este arbitrio sólo podía constituir una solución pasajera, ya que el desequilibrio entre ingresos y gastos hubo de aumentar a medida que se acentuaba la depresión económica y demográfica en el transcurso de las primeras décadas del siglo xvII. Los concejos, más pronto o más tarde, recurrieron al acotamiento y arrendamiento de una parte de los terrenos comunales. Este fenómeno, ya señalado por los contemporáneos ${ }^{39}$, ha sido corroborado, como puede apreciarse en el cuadro I, por la documentación mesteña: desde 1630, cuando menos, las condenas por adehesamientos y acotamientos constituyeron, con gran diferencia, la primera rúbrica de las multas impuestas por los alcaldes mayores entregadores.

\section{CUADRO I}

Condenas de los alcaldes mayores entregadores en la cañada leonesa. Condenas por constitución de nuevas debesas

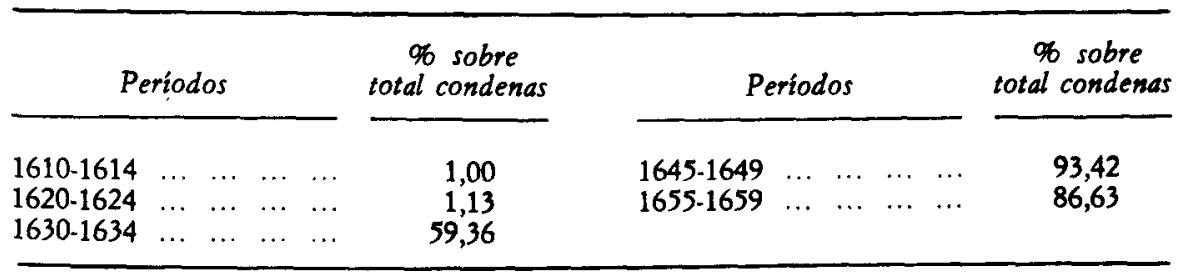

FUENTE: Elaboración propia a partir de las series que aparecen en el trabajo de Fermín MarfN, a quien agradezco la gentileza que tuvo al facilitarme su consulta, La Mesta en los siglos XVI y XVII: cañadas, roturaciones de pastos, arrendamientos e impedimentos de paso y pasto, trabajo inédito que próximamente será presentado como memoria de tesis doctoral en el Departamento de Historia Moderna de la Universidad Complutense de Madrid.

Conviene puntualizar que la Mesta era partidaria de que se produjesen adehesamientos en las zonas donde sus rebaños solian sustentarse durante los inviernos y los veranos, pero se oponía a la reducción de pastos comunales en las zonas de simple tránsito de los ganados trashumantes. Caxa de Leruela expuso con gran claridad la posición de los mesteños sobre los acotamientos: "Aunque es así verdad, que son perjudiciales las dehesas para los ganados

${ }^{39}$ Miguel Caxa de Leruela (1631), pp. 122-129. 
que tienen pasto, paso y aprovechamiento común, y libre, donde se hacen las dehesas, porque se les quita la libertad del aprovechamiento de tal sitio adehesado, no es absolutamente dañoso, ni en todas partes haber hierbas vendibles, porque en las Extremaduras, y en los demás invernaderos son muy convenien. tes, y precisas por causa pública para la conservación de los ganados que bajan de las Sierras para invernar, que como son forasteros, si no se hallaran hierbas compradas, nadie los consintiera en sus baldíos» ${ }^{0}$. El mismo motivo aduce para considerar la utilidad de los acotamientos en las montañas de León.

Aparte de las enajenaciones, pignoraciones, acotamientos y arrendamientos de terrenos públicos llevados a cabo al amparo de normas legales, aun cuando frecuentemente los concejos o los comisionados regios actuasen más allá de los límites establecidos por aquéllas, las simples usurpaciones de los "poderosos» también contribuyeron a acelerar el proceso de privatización de
bienes y derechos comunales. La despoblación de algunas aldeas y las ventas
y exenciones de jurisdicciones fas y exenciones de jurisdicciones favorecieron el «asalto» a los patrimonios públicos". El fenómeno de las «composiciones», mediante el que muchas tierras poseídas en precario fueron confirmadas, también deja entrever, como sugiere Antonio Domínguez Ortiz, el alcance de las usurpaciones de terrenos pú-
blicos

En'suma, difícilmente puede cuestionarse la sensible reducción de los aprovechamientos colectivos del terrazgo en la Castilla de las décadas finales del siglo 'xvi y de la primera mitad del xvir. Ello debió suponer un duro golpe para las economías campesinas, que venían registrando crecientes dificultades desde poco después de mediados del Quinientos. La privatización de bienes y derechos comunales hubo de provocar una merma de las «posesiones» de numerosos productores directos. Por otra parte, el progresivo empobrecimiento de éstos debió traducirse en un retroceso de la propiedad campesina; así lo atestiguan los datos aportados por F. Brumont para la Bureba, comarca en la que, desde que se inició la depresión, el porcentaje de labradores y pequeños campesinos tendió a reducirse' y el de jornaleros a incrementarse ${ }^{43}$. Consiguientemente, dos movimientos debieron producirse de manera simultánea durante la deprésión agraria: por un lado, el descenso del tamaño de las explotaciones de muchos productores directos; por otro, la pérdida de importancia de las «posesiones» y propiedades campesinas en favor de los labrantíos arrendados. Esto último implica que las ganancias que para los productores directos se derivaron del descenso de la renta de la tierra pudieron verse par-

Ibidem, pp. 125-126

"Angel García Sanz, pp. 120-122.

4 Antonio Domínguez Ortiz (1984), p. 230.

"3rancis Brumont (1984), p. 226. 
llos hubieron de tomar en arrendamiento como consecuencia del retroceso de sus propiedades y «posesiones». Por tanto, uno de los factores que supuestamente debía alentar la recuperación del agro, la caída de la renta de la tierra, quedó compensado, cuando menos en parte, por la reducción de la labranza efectuada en terrenos de aprovechamiento vecinal.

Si la progresiva privatización de bienes y derechos comunales perjudicó a las labores de numerosos campesinos, aún debieron ser más intensos_los daños que ocasionó dicho proceso a las actividades pecuarias de aquéllos, entre otras razones por la creciente escasez de pastos que habían provocado las roturaciones y el simultáneo crecimiento de las cabañas estantes durante el siglo xvi. Por otro lado, el alto precio de las hierbas constituyó un importante estímulo para que particulares y concejos procediesen al acotamiento de pastizales. Ello contribuye a explicar el retraso en la recuperación de la ganadería estante: aun cuando el descenso de población y del área de superficie cultivada creaban condiciones favorables para el restablecimiento del equilibrio ecológico, los adehesamientos y acotamientos impedían que los campesinos pudiesen disponer de cantidades significativamente mayores de pastos gratuitos donde sustentar a sus reses auxiliares de labranza. De ahí que las pequeñas cabañas de la mayor parte de campesinos no pudiesen comenzar a recomponerse mientras el movimiento depresivo no hubo alcanzado la intensidad suficiente como para hacer posible un apreciable incremento de la superficie de pastos permanentes mediante el retroceso de los cultivos, fenómeno que no parece acaecer hasta los años treinta y cuarenta del Seiscientos, al menos en extensas zonas de Castilla. Consiguientemente, si la escasez de hierbas constituyó un problema crónico para los cultivadores directos, éste tendió a agudizarse a medida que los acotamientos y adehesamientos se intensificaron.

La supuesta alternativa ganadera en la Castilla de la primera mitad del siglo xvir hubo, pues, de quedar restringida a los dueños de explotaciones que no tenían que desembolsar grandes cantidades por la alimentación de sus reses, bien por ser propietarios de dehesas o cotos redondos, bien por tener la oportunidad de sustentar a un elevado número de cabezas en hierbas de aprovechamiento comunal; es más, teniendo en cuenta la trayectoria de los precios relativos, dichos dueños sólo debieron obtener beneficios de carácter extraordinario entre, aproximadamente,' 1615 y 1630.

La parcial sustitución de bueyes por mulas, fenómeno: qụe en el campo castellano parece iniciarse en el transcurso del siglo xvı, fue favorecida por el alza de los requerimientos de fuerza de tracción animal - debido a las roturaciones $y$, en algunos casos, a la necesidad de labrar más intensamente el terrazgo-, por el auge del comercio de productos agrarios, por el aumento de la distancia media de los núcleos habitados a los terrenos de labor y por la 
extensión del viñedo ${ }^{4}$, pero no hay duda que la insuficiencia de pastos comunales constituyó el factor que más contribuyó a dicho cambio. Esta modificación en el tipo de animales que proporcionaban fuerza de tracción obligó a los campesinos a reestructurar sus labores, aumentando la superficie dedicada a la obtención de cereales-pienso en detrimento de los granos panificables. Consiguientemente, la necesidad de sustituir bueyes por mulas determinó un empeoramiento del nivel de vida de muchos productores directos; además, no todos los campesinos estaban en condiciones de adquirir y mantener un par de mulas: era condición necesaria disponer de una superficie mínima de tierras de labor que permitiese conjugar la producción de alimentos para el ganado con la de cereales para el consumo humano. Por tanto, la privatización del usufructo de numerosos bienes y derechos comunales, al reducir de manera significativa los aprovechamientos colectivos de pastos, forzó la parcial sustitución de bueyes por mulas, lo que vino a acentuar el deterioro de la economía de muchos cultivadores directos y a acelerar el proceso de diferenciación del campesinado

Tampoco conviene olvidar el aumento de labores que hubo de exigir el desarrollo del ganado mular. Ello, junto al agotamiento de los suelos por la mantener el área de superficie cultivada en pueblos que ya estaban perdiendo efectivos. Por tanto, la parcial sustitución de bueyes por mulas contribuye a explicar la prosecución de las roturaciones en un contexto depresivo, roturaciones que dificultaban el restablecimiento del adecuado equilibrio entre la-
branza, pastizales y montes.

No hay duda de que se produjeron importantes «reajustes y readaptaciones» espontáneas en la economía y sociedad castellanas desde finales del siglo xvi, pero los sustanciales cambios en el uso y distribución de los recursos agrarios públicos impidieron que aquéllos lograsen evitar un persistente movimiento depresivo merced al pronto restablecimiento de los necesarios equilibrios entre capacidad productiva y población.

En definitiva, la privatización del usufructo de una parte significativa del patrimonio territorial público, alentada por la importante revalorización que habían registrado los recursos agrarios, particularmente las hierbas, y consentida por un Estado que precisaba utilizar cualquier resorte que permitiese un aumento apreciable de las cantidades recaudadas, contribuyó al deterioro de
multitud de economías campesinas cuyo funcionamiento dependía en buena

" Muchas de las nuevas plantaciones de viñedos de finales del siglo XVI y primeras décadas del xvi tienen relación con el aumento de las necesidades de numerario de los cam. oferta y la simultánea caída de la cargas tributarias. El incremento significativo de la vino en el segundo cuarto del siglo xviI. 
medida de las labores y pastos comunales. Por tanto, aun cuando los cambios en la distribución de la renta también debieron perjudicar a los cultivadores directos, la depresión rural de la Castilla del Seiscientos parece ser, ante todo, una crisis productiva muy ligada a los crecientes problemas que tuvieron que afrontar numerosos campesinos para acceder a los recursos agrarios necesarios para su mantenimiento y reproducción, principalmente a los pastos precisos para el sustento de las reses auxiliares de labranza.

Soy consciente de los sesgos e insuficiencias de este breve ensayo. Por un lado, me he ocupado casi exclusivamente del período depresivo, en tanto que apenas he prestado atención a un tema que, aparte de tener un indudable interés en sí, necesariamente ha de abordarse para obtener una visión global de la crisis del Seiscientos: la lenta recuperación posterior. Por otro, a fin de llamar la atención acerca de la incidencia de los cambios en las formas de aprovechamiento del patrimonio territorial público sobre las economías campesinas, he dejado fuera de este estudio temas de suma importancia como, entre otros, las interrelaciones entre la depresión rural y la urbana, la extensión del régimen señorial, la inestabilidad monetaria, las especiales dificultades registradas por los núcleos de población de corto vecindario ${ }^{45}$ y el endeudamiento campesino y la creciente importancia del crédito usurario.

Quisiera concluir dedicando unas cuantas líneas a una cuestión polémica. ¿Hubo una reacción señorial triunfante en la Castilla del siglo xvir? Es cierto que la nobleza evitó, de facto, el establecimiento de impuestos sobre la riqueza y que los ingresos señoriales, en algunos casos, parecen aumentar. Sin embargo, fueron los «poderosos» locales y parte de la burguesía urbana, el sector que supo reorientar sus actividades cuando se inició la depresión de las ciudades, los principales beneficiarios de la privatización de bienes y derechos comunales y de las ventas de jurisdicciones. En consecuencia, aun cuando la capacidad de integración de la nobleza seguía siendo muy grande, el reforzamiento de las oligarquías locales hubo de exigir cambios en el reparto del poder, sobre todo en el ámbito económico y en el social, que, presumiblemente, recortaron el margen de maniobra de aquélla. Además, las convulsiones que registró el sistema agrario castellano tuvieron que hacer necesaria la readaptación de todas las explotaciones, reto que la «vieja» nobleza, debido, principalmente, a problemas financieros y de gestión, no siempre superó con brillantez. En suma, aun cuando no puede negarse que bastantes economías nobiliarias se enriquecieron y que la mayoría de ellas superaron la depresión del siglo xvir sin graves descalabros, considero que se introduce un sesgo cuando se presenta la «triunfante reacción señorial» como síntesis de la evolución económica y

45 Esta última cuestión fue planteada por Antonio Domínguez Ortiz en un precoz y magnífico artículo (Antonio Domínguez Ortiz (1948), pp. 99-125). 
social de la Castilla de la primera mitad del Seiscientos. La complejidad de los cambios y el estado de las investigaciones aconsejan el empleo de frases menos «redondas» a la hora de concluir.

\section{BIBLIOGRAFIA CITADA}

ANEs, Gonzalo (1978 a): «La "depresión” agraria durante el siglo xvil en Castilla», en Homenaje a Julio Caro Baroja, Madrid.

- $(1978 b)$ : «Tendencias de la producción agrícola en tierras de la Corona de Castilla (siglos XVI a XIX)», en Hacienda Pública Española, núm. 55.

- (1979): «Comercio de productos y distribución de rentas», en ANEs, Gonzalo; Bernal, Antonio; García Fernández, Jesús; Giralt, Emilio, y otros: La economia agraria en la Historia de España. Propiedad, explotación, comercialización, rentas, Madrid.

- (1984): «El sector agrario en la España Moderna», en Papeles de Economia, núm. 20.

Brumont, Francis (1984): Campo y campesinos de Castilla la Vieja en tiempos de Felipe $I I$, Madrid.

Bustelo, Francisco (1972): «Algunas reflexiones sobre la población española de principios del siglo xviII», en Anales de Economía, núm. 15.

CaXa de Leruela, Miguel (1631): Restauración de la antigua abundancia de España, Nápoles.

Do mínGUez ORTIZ, Antonio (1948): «La ruina de la aldea castellana», en Revista Inter. nacional de Sociología.

- (1984): Politica fiscal y cambio social en la España del siglo XVII, Madrid.

Domínguez Ortiz, Antonio, y Vincent, Bernard (1978): Historia de los moriscos. Vida $y$ tragedia de una minoría, Madrid.

García Sanz, Angel (1977): Desarrollo y crisis del Antiguo Régimen en Castilla la Vieja. Economia y sociedad en tierras de Segovia, 1500-1814, Madrid.

- (1980): «Bienes y derechos comunales y el proceso de su privatización en Castilla durante los siglos xvi y xVII: el caso de las tierras de Segoviam, en Hispania, núm. 144.

Hamilton, Earl J. (1934): American Treasure and the price revolution in Spain, 15011650, Cambridge (Mass.); utilizo la versión en castellano, Barcelona, 1975.

- (1947): War and Prices in Spain, 1651-1800, Cambridge (Mass.).

IsRAEL, Jonathan I. (1980): «Spanish Wool Exports and the European Economy, 16101640», en The Economic History Review, 2." ed., vol. XXXIII, núm. 2.

Le Flem, Jean-Paul (1972): "Las cuentas de la Mesta (1510-1709)», en Moneda y Crédito, núm. 121.

López Salazar, Jerónimo, y Martín Galán, Manuel (1981): «La producción cerealista en el Arzobispado de Toledo, 1463-1699", en Cuadernos de Historia Moderna y Contemporánea, 2, Universidad Complutense de Madrid.

LlopIS, Enrique (1980): «Crisis y recuperación de las explotaciones trashumantes: la cabaña del Monasterio de Guadalupe (1579-1679)», en Investigaciones Económicas. núm. 13.

Marcos Martín, Alberto (1985): Economía, sociedad y pobreza en Castilla: Palencia, 1550-1814 (2 tomos), Palencia.

Marín, Fermín (1985): La Mesta en los siglos XVI y XVII: cañadas, roturaciones de pastos, arrendamientos e impedimentos de paso y pasto, trabajo inédito que será presentado como memoria de tesis doctoral en el Departamento de Historia Moderna de la Universidad Complutense de Madrid. 
NadAl, Jordi (1966): La población española (siglos XVI a XX), Barcelona; utilizo la 2." ed., 1971.

Pérez Moreda, Vicente (1978): «La transhumance estivale des merinos de Segovie: le "pleito de la montaña" ", en Melanges de la Casa de Velázquez, tomo XIV.

- (1980): Las crisis de mortalidad en la España interior (siglos XVI-XIX), Madrid.

Ruiz Martín, Felipe (1978): «Procedimientos crediticios para la recaudación de los tributos fiscales en las ciudades castellanas durante los siglos XVI y xVII: el caso de Valladolid", en OTAzu, Alfonso (ed.): Dinero y Crédito (siglos XVI al XIX), Madrid.

VASSBERG, David E. (1983): La venta de tierras baldías. El comunitarismo agrario y la Corona de Castilla durante el siglo XVI, Madrid.

WEISSER, Michael R. (1973): "The decline of Castila revisited: the case of Toledo", en The Journal of European Economic History, 2, 3, pp. 614-640. 